\title{
岸坡地貌发育中耦合均衡理论 的基本特征和意义
}

\author{
崔承琦印萍 \\ （春出海洋大学、青出 266003）
}

提要该理论模式将湖、海岸坡上的波场冲积物宜于波招和望力场的期合作用中。该区带

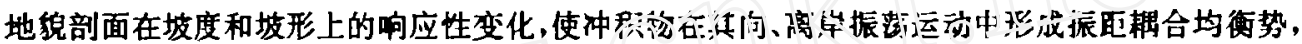

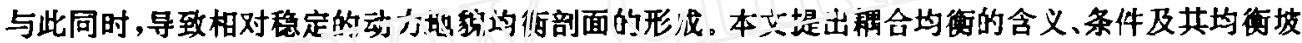

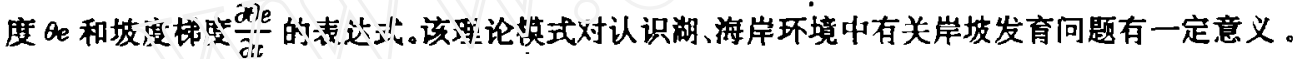

\section{关错词冲积物岸坡 波场一重力场耦合 地貌均衡剖面}

岸坡发育中的均德剖面问题为各国有关学者所广论讨论。由具有一定粒度的冲积物 构成的岸坡在地貌动态上是各种各样的，可以归纳为冲积物上移堆进型和下移蚀退型两类。 如果只考虑到岸坡重力场的作用，在振荡水体作用下，只能有冲积物的下移过程，所有岸坡 圴为虻退型; 如果无枧重力场的影响，只考虑到茾区不对称波的向岸净波场力的作用，那么 只有冲积物的上移过程，所有岸坡都应是堆进型的。以上二种状况无论是在时间上还是在空 间上都是不符合自然界实际的。自然界的实际是在某些时空范围里岸坡动态处于堆进状态， 而在另一些时空范围里岸坡处于虻退状态。除此之外, 人们还注意到, 无论是蚀退抑或堆进 过程都不是无度无限制的。两种过程发育到某种限度之后，在一定的时空范囿里会达到某种 相对稳定的地貌状态。当外界条件发生变化,它又开始新一轮适应于新条件的堆进和冲蚀过 程,而后趋向新的均衡，等等。

早在上世纪一些水工学家和地质学家就通过观察、实验相继提出了岸区浅水波质点运 动的不对称问题，并用于该区泥沙运动的研究，如 A.R. Emy（1831）、E. Beaumout (1845)、 P. Cornaglia (1891) 等; 本世纪至今,一些地质地貌学者,如 N. M. Fenneman (1902)、B. П. Зенкович (1946, 1982)、A. T. Ipean 和 P. S. Eagleson (1955)、W. A. Price (1969)、I. O. Leont ev (1985)等,把其中的有关理论从不同的侧重点应用到岸坡地貌的发育上来; 近些年 来,我国有些学者对岸坡的均衡发育问题也作了有意义的讨论 ${ }^{[1 \sim 5]}$ 。

在这些研究工作中,虽侧重点有所不同,但较一致地认为,在一定的时空范盽里岸坡的 动力均衡过程是确实存在、符合实际的。由于这个地带影响因素的多元化和复杂化,均都做

(1) 冲积物一词在此系指海岸带在波场中运动的推移质和一部分稆移质，是河流冲积物的借用词，通用于海岸，下 同。

收稿日期: 1994 年 1 月 5 日、接受日期: 1994 年 4 月 8 日。

作者简介: 崔承埼, 1939 年生,教投。1962 年南京大学地理系毕业,1965 年该校海洋地敫与海洋地质专业研究生 半业。 


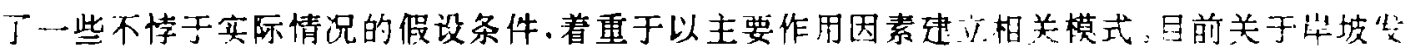
育的均衡理论已成为湖、海岸地貌学基础理论之一, 它勾划出了岸坡地貌发育的基本框架. 并为对此认识的完善和深化提供了正确的基础和广阔的前途.

\section{1 基本特征和意义}

该理论的适用条件是:草坡由波场冲积物(机械组行主要为大于 $0,05 \mathrm{~mm}$. 粗粉砂粒级

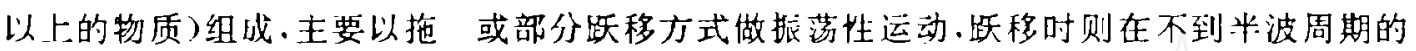

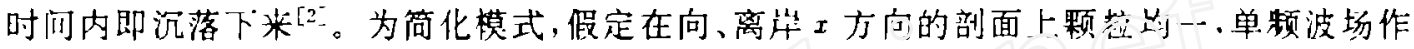

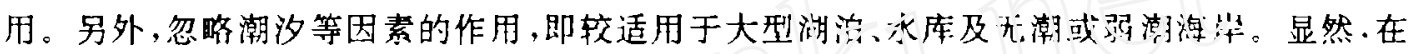

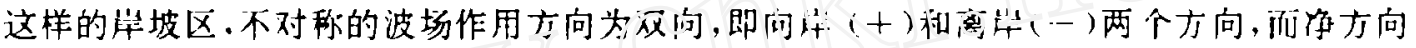

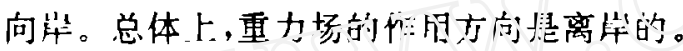

在一定的原治边界条件下，有讨波场的你作用大于重力场的作用（如原始坡度 $\theta$ 较小， 在一个波周期中冲积物在振荡运动中将获得一个向坚的鿇移距 ( $\left.3 S_{*}^{+}\right)$; 相反. 如果波场的净 作用小于重力场的作用 (如原始岸坡的 $\theta$ 较大), 则在一个波周期中. 冲积物在振荡运动中将

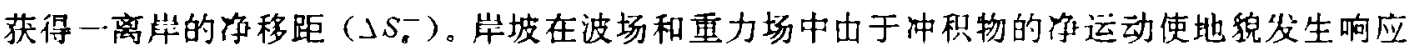
性的变化。在 $د S_{*}^{+}$的状况下, 响应性变化过程是冲积物上移堆积加大岸坡坡度 $\theta$ : 在 $\Delta S_{*}^{-}$的 状况下, 响应性变化过程是冲积物下移堆积在坡脚附近从而降低位坡坡度 $\theta, \theta$ 是重力沿坡 分力的正相关函数。所以不论是在上述哪一种过程中,总是嵌坡通过其地貌坡度的变化使其 上的冲积物向 $\Delta S_{*}^{ \pm} \rightarrow 0$ 的方向发展。有人称之为向“中立点”或“净移零点”方向发展. 即向 岸坡动力均衡方向发展。过程的实质在于地貌坡度的响应性变化是波场和重力耦合的结果, 其地貌均衡是两场耦合均御, 本文称之为岸坡发育的耦合均衡理论。一般开“ $T-T^{-}$”曲线描述 这个过程(图 1)。

图中, $r^{+} 、 V^{-}$你别为近底面水质点向岸和

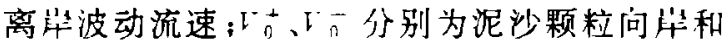
离丝的起动流速: $t_{1} 、 t_{2} 、 t_{3} 、 t_{4}$ 分别为泥沙颗粒向 岸和离坚的起、止动时刻。

斯托克斯二阶波理论对波动过程的描述比 较接近于不对称的浅水波,对此有:

$$
v_{\text {w }}=A \cos w t+B \cos 2 w t
$$

其中, $A 、 B$ 是与波要素和水深有关的系数, $w$ 为 波频 $(2 \pi / T), T$ 为周期。

泥沙的起动流速问题, 在地貌学中有学 者 ${ }^{[2.6-9}$ 采用:

$$
\nu_{0}^{ \pm}=K \cdot \sqrt{d \frac{\sin (\varphi \pm \theta)}{\cos \theta}}
$$

式中, $K$ 为系数, $d$ 为泥沙粒径, $\varphi$ 为自然休止

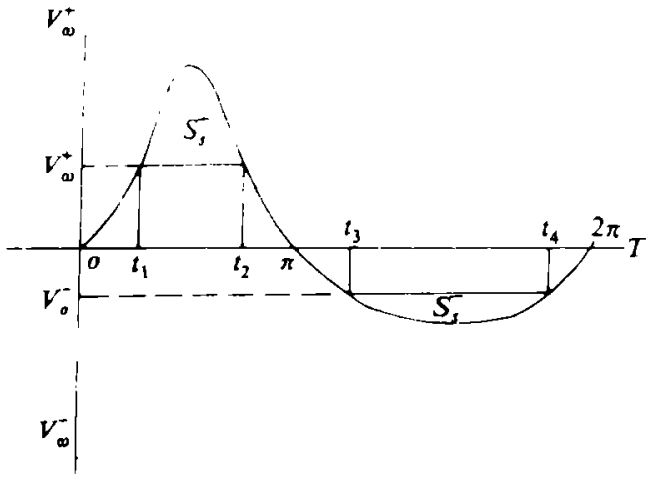

图 1 在一个波周期中近底面波动水质点 和冲积物颗粒运动特佂的 $T-V$ 曲线

Fig. 1 Characteristic $T-I^{\circ}$ curve of bottom wave water particle and fluvial motion in a wave period 
角, $\theta$ 为岸坡平均坡度。

在本文中采用更为广泛的起动流速公式 ${ }^{[5,7]}$;

$$
V_{0}=\sqrt{6.25 a g d}
$$

式中, $a=\left(\rho_{s}-\rho_{0}\right) / \rho_{0}, \rho_{s}$ 和 $\rho_{0}$ 分别为泥沙和水的密度, $g$ 为重力加速度, $d$ 为粒径。

泥沙颗粒起动后的移动速度 $V$ 。为:

$$
V_{s}=V_{w}-f V_{0}
$$

式中, $f=\sqrt{\frac{0.213 \operatorname{tg} \varphi}{C_{d}+C_{l} \operatorname{tg} \varphi}}(f<1.0), C_{d}$ 和 $C_{l}$ 分别是与雷诺数有关的拖 系数和上举力系数。 在海岸地貌中一般取 $f \simeq 1$, 则:

$$
v_{s} \simeq v_{\infty}-v_{0}
$$

如作为矢量, 向岸 为“+”, 离岸为“-”, 则:

$$
V \pm=V \pm-V \pm
$$

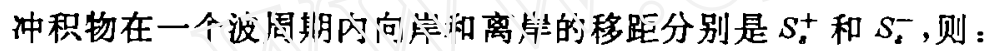

$$
\begin{aligned}
& S_{t}^{+}=\int_{t_{1}}^{r_{2}}\left(V_{t}^{+}-V_{0}^{+}\right) \mathrm{d} t \\
& S_{*}^{-}=\int_{t_{3}}^{P_{4}}\left(V_{*}^{-}-V_{0}^{-}\right) \mathrm{d} t
\end{aligned}
$$

岸坡冲积物在重力场沿坡分力作用和起动的条件下，半波周期内的下移距以 $s_{g}$ 表示:

$$
S_{0}=\frac{1}{8} g T^{2} \sin \theta
$$

似据上述关系，建立了在任何波向线的情况下冲积物在岸坡上移距的矢量图 (图 2)。

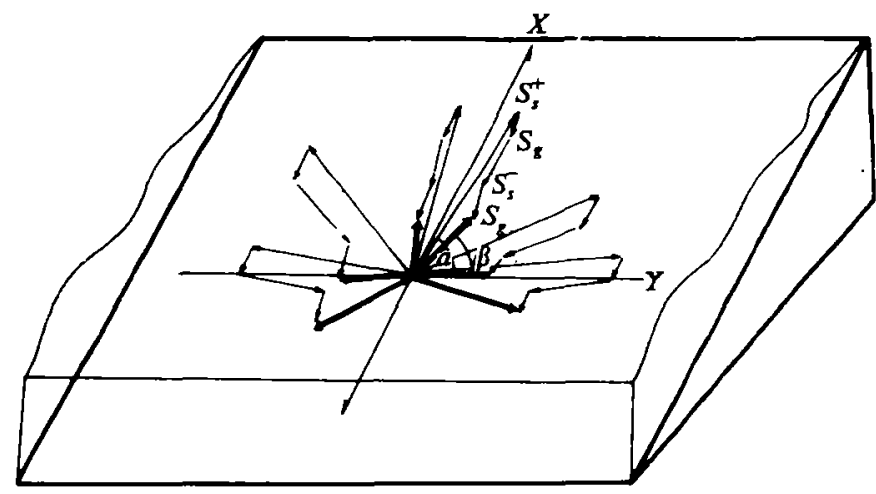

图 2 冲积物在岸坡工移臤矢量图

Fig. 2 Vectograph of sediments in submarine slope

根据湖、海岸描述习惯, 图示中 $y$ 轴为沿等深线方向, $x$ 轴为垂直于岸方向, $a$ 为波向线

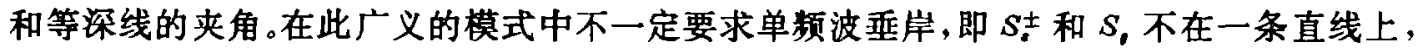
波向线垂直于岸的情况 $(a=\pi / 2)$ 只是其中的特例。矢量以 $\Delta S_{s}$ 以“ $\Rightarrow$ ”表示, 它代表在一个 波周期内冲积物移动的净矢距, 显然: 


$$
\Delta S_{d}= \pm\left[\left(S_{a}^{+}-S_{s}^{-}\right)^{2}-4 S_{g}\left(S_{q}^{+}-S_{a}^{-}\right) \sin \alpha+4 S_{q}^{2}\right]^{1 / 2}
$$

$\Delta \mathrm{S}$, 与 $\mathrm{x}$ 方向的夹角为 $\beta$ :

$$
\beta \simeq \operatorname{arctg}\left[\operatorname{tg} a-\frac{2 S_{I}}{\left(S_{a}^{+}-S_{a}^{-}\right) \cos \alpha}\right]
$$

$\Delta S$ 。的垂岸分量 :

$$
\Delta S_{\varepsilon}=\Delta S_{s} \sin \beta
$$

$\Delta S_{a}$ 的沿岸分量：

$$
\Delta S_{a}=\Delta S_{s} \cos \beta
$$

在冲积物的不断振荡中剖面上, 每点动力地貌耦合均微的意义在于:

$$
\Delta S_{z}=\Delta S_{q} \sin \beta=0
$$

说明均衡时 $\beta=0$ 或 $\beta=\pi$, 而不取妜于 $\Delta S$ 能大小，动

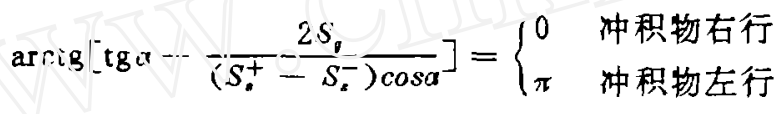

$$
\begin{aligned}
& S_{\mathrm{g}}=\frac{\left(S_{t}^{+}-S_{c}^{-}\right)}{2} \sin a=0
\end{aligned}
$$

在这个模式中，表征波场对冲积物净移距作用大小的指标是 $\left(S_{*}^{+}-S_{*}^{-}\right)$, 这与波场向、 离岸的不对称指标是等价的; 表征重力场对冲积物移距作用大小的指标是 $S_{g}$, 并与坡度 $\theta$ 为 正相关函数, $S_{\mathbf{o}}=f(\theta)$ 。岸坡剖面地貌形态的变化, 即响应性、调整性变化是通过改变其坡 度 $\theta$ (从而改变 $S_{q}$ ) 的大小表现出来的。如果坡角 $\theta$ 过小,也即 $S_{q}$ 过小, $0<\beta<\pi$, 则:

$$
S_{0}<\frac{\left(S_{a}^{+}-S_{a}^{-}\right)}{2} \sin a
$$

这种条件下冲积物上移堆积, 逐渐加大 $\theta$, 即加大 $S_{\mathrm{g}}$; 如果坡角 $\theta$ 过大, 也即 $S$, 过大, $\pi<$ $\beta<2 \pi$, 则 :

$$
S_{0}>\frac{\left(S_{*}^{+}-S_{\varepsilon}^{-}\right)}{2} \sin a
$$

这种条件下冲积物下移向坡脚堆积, 逐渐降低 $\theta$, 即减小 $S_{g}$ 。以上两个过程势必都向耦 合均衡方向发展, 如达到 $\beta=0$ 或 $\beta=\pi$, 也即 $S_{g}=\frac{\left(S_{*}^{+}-S_{a}^{-}\right)}{2} \sin \alpha$ 时, 即达到在这种时空条 件下的耦合均衡。这是一种相对的动力地貌稳定状态。根据式 (9)、(15) 及其等价表达式, 则 该处的均衡坡度为:

$$
\theta e=\arcsin \left[\frac{4\left(S_{s}^{+}-S_{s}^{+}\right)}{g T^{2}} \sin a\right]
$$

在某一定 $a$ 值条件下, 令 $\varphi=4 \sin a / g T^{2}$, 显然 $\varphi$ 为常数,则有：

$$
\frac{\partial \theta e}{\partial x}=R \frac{\partial\left(S_{t}^{+}-S_{s}^{-}\right)}{\partial x}
$$

式中,系数 $R=\varphi / \sqrt{1-\left[\phi\left(S_{s}^{+}-S_{s}^{-}\right)\right]^{2}}$, 根据自然性征, $R>0$ 。

式 (20) 左部 $\frac{\partial \theta e}{\partial x}$ 为岸坡在垂直方向上的坡度梯度, 即坡形指标; 右部 $\frac{\partial\left(S_{a}^{+}-S_{a}^{-}\right)}{\partial x}$ 为岸 坡上冲积物在垂岸方向上的振荡运距的不对称梯度, 是波场作用的不对称梯度指标。表达式 
(20) 有以下二个意义:第一,岸坡的耦合均衡地貌剖面的坡度梯度 $\frac{\partial \theta e}{\partial x}$ 与波场的不对称梯度 $\frac{\partial\left(S_{s}^{+}-S_{s}^{-}\right)}{\partial x}$ 是一致的; 第二,在自然状况下 $\left(S_{*}^{+}-S_{s}^{-}\right)$的变率一般都是正值, 也决定了 $\frac{\partial \theta e}{\partial x}$ 为正值, 也即耦合均得剖面一般是上凹型坡。但也应指出, 在向海射流的物源区,如河口三角 洲河滩区, $\left(S_{s}^{+}-S_{s}^{-}\right)$的变率也可能是负值,这样也可以形成一种上凸形坡。

此外,耦合均得理论还可以揭示岸坡地貌动态方面的其它一些过程和现象。自然界的一 个岸区, 长期平均状况, $\left(S_{s}^{+}-S_{s}^{-}\right)$变化幅度是不大的, 但在短期内由于风场的变化, 会引起 波场结构相当大的变化。在比较对称的岸区风浪作用下, $S_{*}^{+} \simeq S_{s}^{-}$, 据表达式 $(10), \Delta S_{8} \simeq$ $2 S_{p}, \beta=\frac{3}{2} \pi$ 。说明在这种条件下，岸坡上的冲积物基本:上做净离岸问下仿移动，湖、海岸表

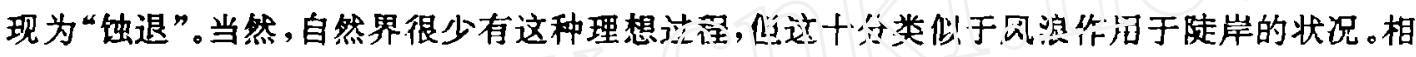

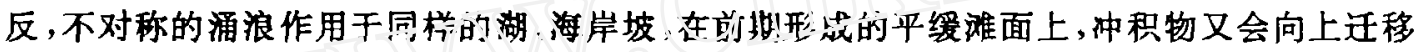

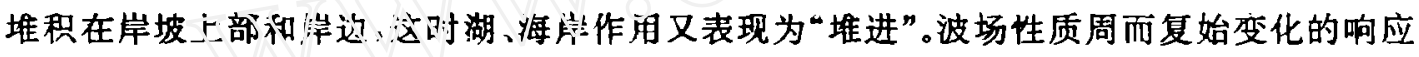
过程，导致湖、溹:岸的沉积地貌循环。另外，相对于某段湖、海岸的波向，长期平均状况，虽然 没有大幅度变化, 但在短时间内却有相当大的变化, 这会导致 $a$ 角的变化。根据该理论, 在波 场作用不对称指标相同的条件下, $a$ 角与岸坡均衡坡度 $\theta e$ 为正相关关系，即近垂向波作用期 间岸坡向加陡的方向发育，而斜向波作用期间岸坡又向平缓方向发育。在同一波向期，走向 不同的各个岸坡地段,波向线与各段湖、海岸的夹角有大有小。同理， $a$ 角较大的岸段，经过 一段作用以后,会比 $a$ 角较小的岸段的岸坡坡度大。

\section{2 结 论}

岸坡剖面的动力地貌均衡是该地带波场和重力场对冲积物作用的耦合均衡。岸坡向耦 合均衡发育的过程即岸坡通过其坡度和坡形变化的响应过程。耦合均衡动力地貌状态的概 要表达式是 $\Delta S_{\alpha}=\Delta S_{s} \sin \beta=0$ 。岸坡地貌是该地带自然环境重要环节之一, 其动态与形态 特征,对于这一地带环境的研究具有一定的理论和实践意义。

\section{参 考 献}

1 Komar. P D 海谁过程与沉积作用。北京: 海洋出版社,1985. 129 142

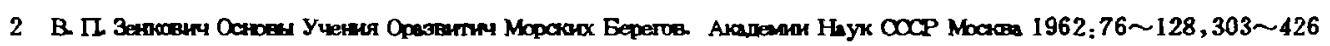

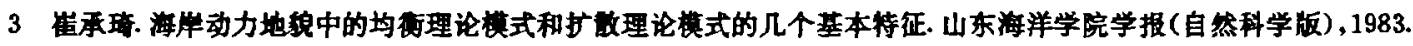
13(3)

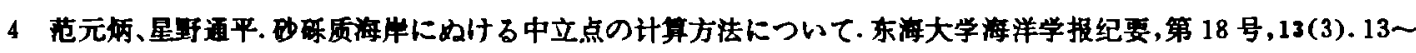
14

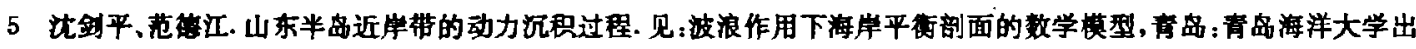
版社, 1992. 104 136

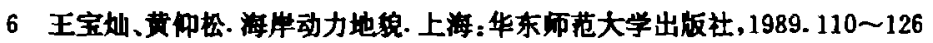

7 空国化. 论记沙起动流速. 水利学报, 1960,4.44 60 
8 Leonjev I O. Sediment transport and beach equiliórium profile, Coastal $B_{n g}$. , 1985,9. 277 291

\title{
BASIC CHARACTERISTICS AND SIGNIFICANCE OF COUPLED EQUILIBRIUM THEORY IN SUBM ARINE SLOPE MORPHOLOGIC DEVELOPMENT
}

\author{
Cui Chengqi Yin Ping \\ (Ocean University of Qingdao, Qingdao 266003)
}

\begin{abstract}
Abstreat
The sediments moving on-stf shore in wave fieid on leise sea submarine slope are coupled by wave and gravity field. The sel: on oif shore displacement of the sediments leads to the changing of subinarime morphological profile in slope and the gradient of slope. This is a morphological response process to external agent. The condition of coupling equilibrium profile is :

$$
\begin{aligned}
& \Delta S_{\infty}=\Delta S_{\text {s }} \cos \beta=0 \\
& \Delta S_{\mathrm{s}}= \pm\left[\left(S_{\mathrm{s}}^{+}-S_{\mathrm{s}}^{-}\right)^{2}-4 S_{\mathrm{s}}\left(S_{\mathrm{z}}^{+}-S_{\mathrm{s}}^{-}\right) \sin a+4 S_{\mathrm{f}}{ }^{2}\right]^{1 / 2} \\
& \beta=\operatorname{arctg}\left[\operatorname{tg} \alpha-\frac{2 S_{q}}{\left(S_{a}^{+}-S_{a}^{-}\right) \cos \alpha}\right]
\end{aligned}
$$

There is an approximate relation between gradient of the slope in equilibrium $(\theta e)$ and asymmetrical gradient of the oscillating motion of sediments along the submarine shore slope.

$$
\frac{\partial \theta e}{\partial x}=\frac{\partial\left(S_{+}^{+}-S_{+}^{-}\right)}{\partial x} \cdot R
$$

where, $\Delta S_{s} \longrightarrow$ net displacement of sediment in a period of wave;

$\Delta S_{\text {ar }} \longrightarrow$ net displacement of sediment in a period of wave in the direction perpendicular to shoreline;

$\beta-$ angle between displacement vector of sediment and the shoreline in a period of wave;

$a-$ angle between incident wave and shoreline;

$S_{\mathrm{b}} \longrightarrow$ distance of sediments displacement caused by the gravity along the slope in half period of wave ;

$S_{a}^{+} \longrightarrow$ distance of onshore displacement of sediment caused by wave;

$S_{*}^{-}-$distance of offshore displacement of sediment caused by wave;

$R-$ coefficient $(>0)$
\end{abstract}

Key Words Submarine slope, couple fields, geomorpholog ical equilibrium 